\title{
Preparation and analysis of zero gases for the measurement of trace VOCs in air monitoring
}

\author{
Jennifer Englert ${ }^{1}$, Anja Claude ${ }^{1}$, Alessia Demichelis ${ }^{2}$, Stefan Persijn ${ }^{3}$, Annarita Baldan ${ }^{3}$, Jianrong Li $^{3}$, \\ Christian Plass-Duelmer ${ }^{1}$, Katja Michl ${ }^{1}$, Erasmus Tensing ${ }^{1}$, Rina Wortman $^{3}$, Yousra Ghorafi ${ }^{3}$, Maricarmen Lecuna ${ }^{4}$, \\ Guido Sassi $^{4}$, Maria Paola Sassi ${ }^{2}$, and Dagmar Kubistin ${ }^{1}$ \\ ${ }^{1}$ Deutscher Wetterdienst (DWD), 82383 Hohenpeissenberg, Germany \\ ${ }^{2}$ Istituto Nazionale di Ricerca Metrologica (INRIM), 10135 Torino, Italy \\ ${ }^{3}$ VSL - Dutch Metrology Institute, 2629 JA Delft, the Netherlands \\ ${ }^{4}$ Politecnico di Torino (POLITO), 10135 Torino, Italy
}

Correspondence: Anja Claude (anja.claude@dwd.de) and Dagmar Kubistin (dagmar.kubistin@dwd.de)

Received: 16 November 2017 - Discussion started: 28 November 2017

Revised: 5 April 2018 - Accepted: 18 April 2018 - Published: 4 June 2018

\begin{abstract}
Air quality observations are performed globally to monitor the status of the atmosphere and its level of pollution and to assess mitigation strategies. Regulations of air quality monitoring programmes in various countries demand high-precision measurements for harmful substances often at low trace concentrations. These requirements can only be achieved by using high-quality calibration gases including high-purity zero gas. For volatile organic compound (VOC) observations, zero gas is defined as being hydrocarbon-free and can be, for example, purified air, nitrogen or helium. It is essential for the characterisation of the measurement devices and procedures, for instrument operation as well as for calibrations. Two commercial and one self-built gas purifiers were tested for their VOC removal efficiency following a standardised procedure. The tested gas purifiers included one adsorption cartridge with an inorganic media and two types of metal catalysts. A large range of VOCs were investigated, including the most abundant species typically measured at air monitoring stations. Both catalysts were able to remove a large range of VOCs whilst the tested adsorption cartridge was not suitable to remove light compounds up to $\mathrm{C}_{4}$. Memory effects occurred for the adsorption cartridge when exposed to higher concentration. This study emphasises the importance of explicitly examining a gas purifier for its intended application before applying it in the field.
\end{abstract}

\section{Introduction}

Volatile organic compounds (VOCs) play an important role in atmospheric chemistry. They are key substances in the tropospheric ozone and secondary organic aerosol formation, affecting human health and climate. The main sources of VOCs are biogenic processes (e.g. plant metabolism) and anthropogenic activities (e.g. fossil fuel or industrial solvents emissions). The variety of VOCs is enhanced by subsequent oxidation processes. The main sink process is the oxidation by the daytime cleaning agent, the hydroxyl radical $(\mathrm{OH})$. Thus, the abundance of VOCs alters the self-cleaning capacity of the atmosphere and the removal of less reactive pollutants like carbon monoxide and the greenhouse gas methane.

VOC concentrations in the background atmosphere are typically at low levels of a few pmol mol${ }^{-1}$ up to some nmol mol ${ }^{-1}$, demanding measurement techniques with very high sensitivities, e.g. gas chromatography (GC) systems or state-of-the-art proton-transfer-reaction mass spectrometers (PTR-MS). High-quality zero gases are needed for determining their background signals and for performing system checks, e.g. blank, memory effect and leak detections. Additionally, zero gases are essential for dynamic calibration methods since VOC calibration standards are often generated either by permeation or diffusion into a controlled zero gas stream (ISO6145-10, 2002; ISO6145-8, 2005; Demichelis et al., 2016). An alternative is the dynamic dilution of a highly concentrated static standard gas mixture with a zero gas stream using mass flow controllers (ISO6145-7, 2009). 
Besides, zero gases are applied for the operation of GC systems as carrier gas of GC columns and for fuel gas of flame ionisation detectors. The need for high-purity zero gases is further driven by more stringent quality objectives from the WMO GAW programme (WMO, 2007) or the ACTRIS network (Hoerger et al., 2015; ACTRIS, 2014). These networks aim to observe the long-term trends of VOC concentrations in the background atmosphere. Though, few studies with a detailed characterisation of the performance of gas purifiers have been conducted so far (e.g. Miñarro et al., 2016; Haerri, 2009).

A high-quality zero gas is defined by containing insignificant concentrations of the target components to be measured. In particular for VOC measurements, the hydrocarbon compounds of the zero gas have to be below the detection limit of the instruments. The highest-quality commercial zero gases in gas cylinders (air, nitrogen or helium grade 5.5 or higher) are specified to contain below 10 to $100 \mathrm{nmol} \mathrm{mol}^{-1}$ total hydrocarbons. These levels far exceed the needed purity for a zero gas in atmospheric background monitoring with concentrations down to some $\mathrm{pmol} \mathrm{mol}^{-1}$. To reduce the amount fraction of VOCs, different gas purification technologies are available. Preparation has to be simple, fast, low cost and applicable at remote unattended stations. Furthermore, the preferred method is dependent on the VOCs present in the gas to be purified, the gas matrix and maintenance interval. Commonly used purification technologies in atmospheric monitoring include, but are not limited to, gas purifiers based on inorganic media (e.g. Conte et al., 2008) or activated carbon (Van Osdell et al., 1996; Sircar et al., 1996), metal catalysts (Liotta, 2010; Heck et al., 2009) and photocatalytic techniques (Debono et al., 2013; Huang et al., 2016).

In this study, three purifiers were selected to test their removal efficiency of a defined amount of VOCs to be applicable for ambient air monitoring stations. An adsorption cartridge with an inorganic media was selected for low-cost zero gas production without the need for electricity. In addition, the commonly used catalytic technique with an infinite lifespan has been tested for two types of catalyst.

\section{Experimental}

\subsection{Tested purifiers and analytical methods}

The tested commercial adsorption cartridge was based on inorganic media not being further specified by the manufacturer (it was agreed not to publish the name and trademark). Clean dry air (CDA) was stated to be used as the input gas with a maximum flow rate of $50 \mathrm{slpm}$. No additional heating of the purifier was required. The manufacturer claimed the removal of condensable organics below $1 \mathrm{pmol} \mathrm{mol}^{-1}$ without any further specifications of those compounds. Maximal incoming contaminant concentrations were indicated with $10 \mu \mathrm{mol} \mathrm{mol}^{-1}$. The lifetime was stated to be 1 year at nominal flow rate with $1 \mu \mathrm{mol} \mathrm{mol}^{-1}$ inlet challenge of moisture. The second purifier was a commercial catalyst with 3-5\% palladium oxide (manufacturer SAES Pure Gas, model PS15-GC50-CDA-2). It was specified for CDA with a maximum flow rate of $3 \mathrm{slpm}$. Its operation temperature was $350^{\circ} \mathrm{C}$. Elimination of methane and NMHCs below $1000 \mathrm{pmol} \mathrm{mol}^{-1}$ was stated by the manufacturer. Maximum inlet impurities were $2 \mu \mathrm{mol} \mathrm{mol}^{-1}$ total hydrocarbons. At the rated flow of $3 \mathrm{slpm}$ and at rated working temperature the manufacturer stated an infinite lifespan of the catalyst without the need of regeneration. The third purifier was a home-made metal catalyst built by the German Meteorological Service (Deutscher Wetterdienst, DWD). It consisted of a stainless steel tubing ( 1 in. diameter) with a length of $1 \mathrm{~m}$ filled with aluminium oxide pellets with $0.5 \%$ platinum (Heraeus, Germany). The tubing was heated to $400{ }^{\circ} \mathrm{C}$ and was built in an aluminium profile box filled with perlite for thermal insulation. A stainless steel mesh $(25 \mu \mathrm{m})$ at the end of the tubing was used for particle protection of the subsequent instruments.

The performance of the purifiers was tested by detecting residual VOC concentrations in the zero gas with GC systems (Table S1 in the Supplement). Prior to GC analysis VOC fractions were pre-concentrated by either adsorbent materials or cryogenically cooled glass beads. Subsequently, the VOCs were thermally desorbed from these traps and separated into one or more capillary columns of the GC. For detection, flame ionisation detectors or mass spectrometers were deployed. Five different GC systems were used: two for non-methane hydrocarbons (NMHCs) operated by DWD (Hoerger et al., 2015; Plass-Duelmer et al., 2002) and the Dutch Metrology Institute (VSL), one for monoterpenes by DWD (Hoerger et al., 2015) and three for oxygenated VOCs (OVOCs) by DWD, VSL and the Istituto Nazionale di Ricerca Metrologica (INRIM) (Demichelis et al., 2016). A large range of VOCs were investigated, including the most abundant species typically measured at air monitoring stations as well as acetonitrile (see Table 1).

Detection limits for all systems were determined using the IUPAC method based on the Neyman-Pearson theory of hypothesis testing (IUPAC, 1995; Sect. S2 in the Supplement).

\subsection{Experimental measurement set-up and procedure}

For comparability, a common procedure was applied by the three labs. Sample volumes used were dependent on the requirements of the different analysers. In general, sample volumes between 400 and $3000 \mathrm{~mL}$ were applied. For all following steps a repetition of five consecutive runs was recommended:

1. In step 1, the in-house zero gas was measured directly by the analysis systems to quantify its VOC impurities. Additionally, all analysis systems were checked for internal blanks, i.e. system artefacts, and their discrimination from zero gas impurities was done by measuring 


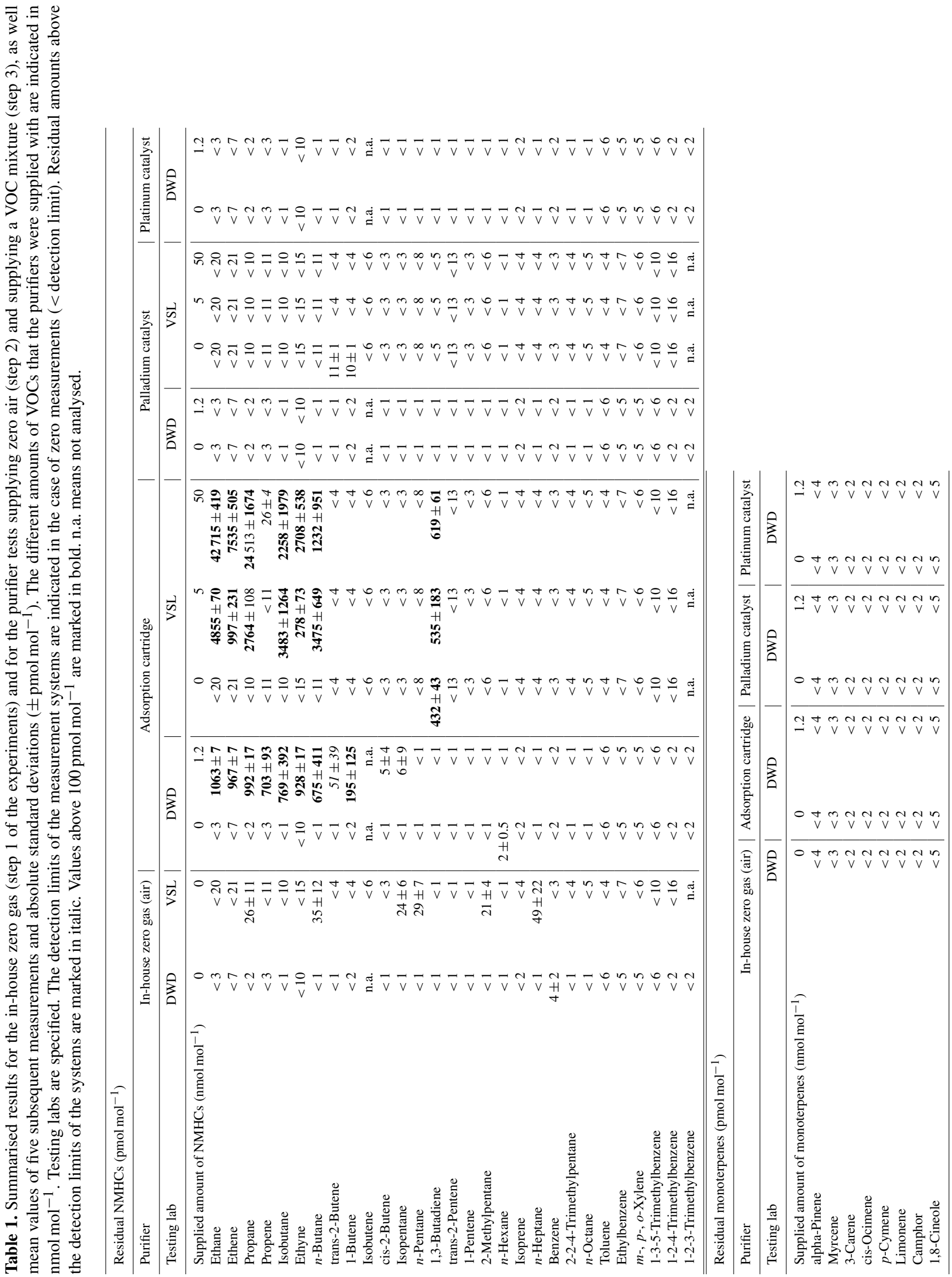




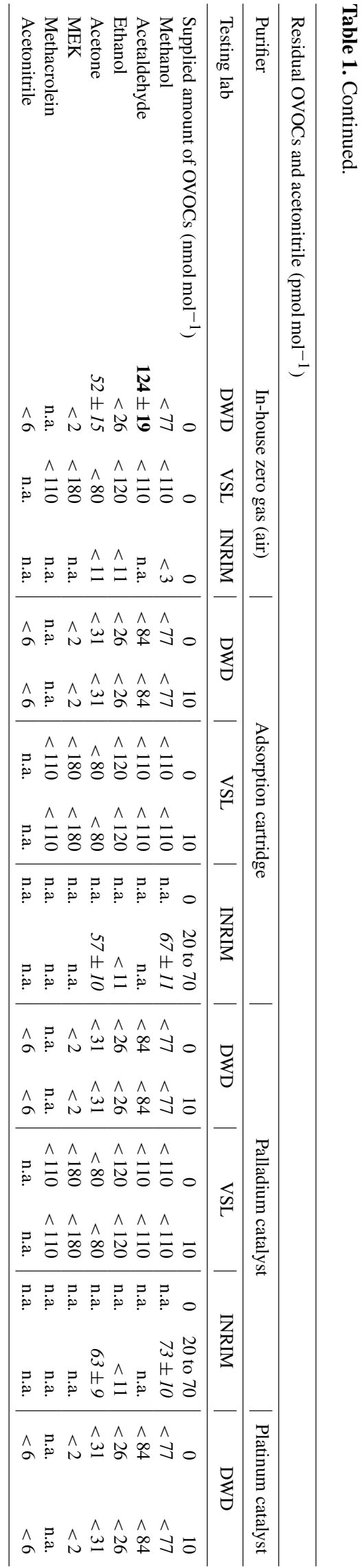

different sample volumes of the in-house zero gas. A proportional relationship of the detector response with the sampled volume is expected for impurities in the inhouse zero gas, whereas for GC system internal blanks the detector response is expected to be independent of the sample volume. The tested in-house zero gas was used for the following steps of the experiment (2 to 4 ).

2. In the next step the in-house zero gas from step 1 was supplied to one specific purifier to quantify the VOC impurities originating from the purifier itself.

3. In the third step the efficiency of VOC removal of the tested purifier was checked by supplying a VOC mixture and measuring the outcome of residual VOCs.

4. In the last step the incoming VOC concentration for step 3 was checked by supplying the same preparation of VOC mixture directly to the analysis system (no purifying).

After step four a repetition of steps one and two was optional for the labs but is advisable to monitor the status of the setup.

A unified flow rate of 1 slpm was applied being within the specification of each purifier model. The two catalysts were heated and flushed with zero gas for at least $2 \mathrm{~h}$ before starting the experiments. This was needed to reduce VOC impurities originating from the catalysts being freshly installed. The experimental set-up is shown in Fig. 1.

Test mixtures with different VOC mole fractions were produced by dynamic generation methods, e.g. dilution of high concentrated static VOC mixtures in cylinders (Fig. 1) or diffusion methods (Demichelis et al., 2016). The following test mixtures were supplied: NMHCs at 1.2, 5 and $50 \mathrm{nmol} \mathrm{mol}^{-1}$; monoterpenes at $1.2 \mathrm{nmol} \mathrm{mol}^{-1}$; OVOCs from 10 to $70 \mathrm{nmol} \mathrm{mol}^{-1}$; and acetonitrile at $10 \mathrm{nmol} \mathrm{mol}^{-1}$. For the in-house zero gas DWD used compressed and dried (water content $\sim 1000 \mu \mathrm{mol} \mathrm{mol}^{-1}$ ) ambient air purified by a palladium catalyst. VSL and INRIM used synthetic air cylinders (grade 6.0, water content $<0.5 \mu \mathrm{mol} \mathrm{mol}^{-1}$, total hydrocarbons content $<0.05 \mu \mathrm{mol} \mathrm{mol}^{-1}$ ).

\section{Results and discussion}

To ensure comparability between the participating groups the same measurement procedure described in Sect. 2.2 has been applied. All gas chromatograms were analysed visually. Peaks of VOCs in the chromatograms were integrated by GC software and mole fractions were subsequently determined for each single measurement and average mole fractions and standard deviations, respectively, were derived for each measurements series (Table 1).

Before assessing the purifier efficiency, in-house zero gas quality and internal blanks were determined by step one of the measurement procedure (Sect. 2.2). The results for 


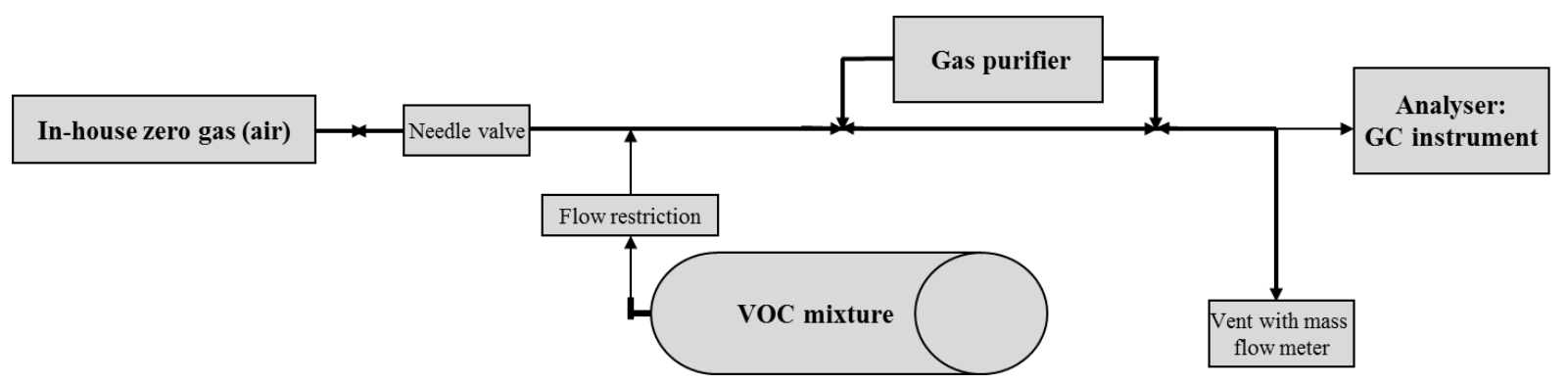

Figure 1. Experimental set-up for testing the purifier performance.

VSL and DWD are shown in the first two columns in Table 1. In the DWD in-house zero air all substances were below the detection limit, with the exception of benzene $\left(4 \mathrm{pmol} \mathrm{mol}^{-1}\right)$, acetaldehyde $\left(124 \mathrm{pmol} \mathrm{mol}^{-1}\right)$ and acetone $\left(52 \mathrm{pmol} \mathrm{mol}^{-1}\right)$. The observed peaks were independent of the sample volume (see Figs. S1 and S2 in the Supplement), showed the characteristics of an internal blank and are not regarded as an impurity of the DWD in-house zero gas. For VSL, blank values were observed at a level of 20$50 \mathrm{pmol} \mathrm{mol}^{-1}$ for several alkanes (Table 1). The results are consistent with the specification of the used synthetic air grade 6.0 allowing up to $50 \mathrm{nmol} \mathrm{mol}^{-1}$ of hydrocarbons. This highlights the need for further purification of commercial cylinders to assure low impurity levels for high-quality zero air. With the INRIM system, which focused on OVOCs only, no blanks were observed in their in-house zero gas. Subsequently, VOC release of the purifier itself was checked (step 2 in Sect. 2.2). For example, the platinum catalyst showed acetaldehyde impurities scaled with the sample volume (Figs. S1 and S3). By flushing the catalysts for $2 \mathrm{~h}$ with zero air $(1 \mathrm{slpm})$, the relevant impurities were below the detection limits.

After characterisation of the blank values and purifier impurities, the purifier efficiencies were determined (step 3 in Sect. 2.2). In Table 1, the results of all labs are summarised. Both tested catalysts (palladium as well as platinum) removed NMHCs and monoterpenes to concentrations below the detection limits which were generally below $10 \mathrm{pmol} \mathrm{mol}^{-1}$.

All tested OVOCs were removed to mole fractions below $100 \mathrm{pmol} \mathrm{mol}^{-1}$ by the tested purifiers. Only the lab of INRIM detected residuals of methanol and acetone above the detection limits of their system. These OVOCs are generally prone to adsorption and desorption effects on surfaces in the instruments and therefore subject to high measurement uncertainties and blank values. Consequently, detection limits are usually elevated as seen for the DWD and VSL systems (Table 1). For the INRIM system, however, rather low detection limits were indicated and no blank values were reported. Nevertheless, the fact that similar mole fractions for methanol and acetone (Table 1) were detected by INRIM for both types of purifiers and for varying input concentrations
(20-70 $\mathrm{nmol} \mathrm{mol}^{-1}$ ) implies the possibility that here system blanks or artefacts were observed. Unfortunately, a repetition of the blank measurements was not performed at INRIM with this set-up after this experiment and no further conclusions can be drawn.

For the adsorption cartridge a breakthrough of light NMHCs (from $\mathrm{C}_{2}$ to $\mathrm{C}_{4}$ ) was observed by all testing labs (Table 1). At a sample flow of $1 \mathrm{slpm}$ ethane, ethene, propane, propene, isobutane, ethyne, $n$-butane, trans-2-butene, 1butene and 1,3-butadiene were not efficiently removed.

Except for ethane, the removal efficiency is not consistent for different input concentrations. For ethene, propane, propene, ethyne, trans-2-butene and 1-butene, the $1.2 \mathrm{nmol} \mathrm{mol}^{-1}$ input was less efficiently purified compared to the higher inputs. Several reasons are possible: first, these results were produced by two different labs which tested the same model of cartridge but not the identical cartridge. The two cartridges may show different behaviours. Furthermore, DWD responsible for the $1.2 \mathrm{nmol} \mathrm{mol}^{-1}$ experiment used a zero gas for the tests which had a much higher humidity (water content $\sim 1000 \mu \mathrm{mol} \mathrm{mol}^{-1}$ ) than the test gas from VSL which came from a commercial synthetic air cylinder (water content $<0.5 \mu \mathrm{mol} \mathrm{mol}^{-1}$ ). The humidity level has an impact on the purifier lifetime. The manufacturer of the adsorption cartridge stated that the humidity of the DWD zero gas would saturate this kind of cartridge almost immediately (personal communication, 2017). It should only be used with very dry air with at maximum $1 \mu \mathrm{mol} \mathrm{mol}^{-1}$ water content. A closer look into the individual results of the measurements series of the VOC mixture running through the adsorption cartridge reveals another effect: the breakthrough behaviour is affected by the repetition of measurements and changes with each iteration (Fig. S6). This is reflected in high standard deviations for some substances in Table 1.

All $\mathrm{C}_{5}$ and heavier NMHCs, monoterpenes and acetonitrile were removed to values below the detection limits of the systems. For OVOCs, see the discussion of the catalyst results above. 


\section{Conclusions}

Two tested catalysts in this study were able to remove a large range of different VOCs. High mole fractions up to $50 \mathrm{nmol} \mathrm{mol}^{-1}$ were purified and residual concentrations were below the detection limits of the systems going down to less than $1 \mathrm{pmol} \mathrm{mol}^{-1}$ for NMHCs.

The tested adsorption cartridge was not suitable to remove light NMHCs $\left(\mathrm{C}_{2}\right.$ to $\left.\mathrm{C}_{4}\right)$. There was a breakthrough behaviour of these compounds which was not constant. Also, VOC memory effects were observed. To characterise these effects, repetition of measurements ( $>5$ ) would be an advantage. However, it removed heavier VOCs, OVOCs and monoterpenes. An advantage of the adsorption cartridge is the lack of electricity. It could be a good alternative for applications where the breakthrough of light VOCs is of no relevance. A big disadvantage is the high influence of humidity on the lifetime of this kind of purifier. The tested model in this study was only adequate for use with very dry air up to maximum $1 \mu \mathrm{mol} \mathrm{mol}^{-1}$ water content. With this awareness it is highly recommended to enquire about the maximum applicable water content of the used gas from the manufacturer of a purifier.

Finally, zero gas is often produced by compression of ambient air, which constitutes a complex matrix with residual humidity. The cleaning process to receive high-purity zero gases is a challenge to any purifying system. It is highly important to explicitly examine a gas purifier for its intended application. Tests should be done at the given conditions, e.g. the same flow rates and the same gas matrix with special focus on given target component concentrations and humidity. For the tests, measurement systems with adequate detection limits are essential. Potential internal blanks have to be detected and well characterised. Their long-term behaviour has to be controlled, especially for the enduring use in air quality monitoring stations.

Data availability. The data set can be made available on request. Please contact anja.claude@dwd.de.

Supplement. The supplement related to this article is available online at: https://doi.org/10.5194/amt-11-3197-2018-supplement.

Competing interests. The authors declare that they have no conflict of interest.

Acknowledgements. The research leading to these results was performed under the European Metrology Research Programme (EMRP) project ENV56 KEY-VOCs, which is jointly funded by the EMRP participating countries within EURAMET and the European Union.
Edited by: Andreas Hofzumahaus

Reviewed by: Jochen Rudolph and one anonymous referee

\section{References}

ACTRIS: WP4-NA4: Trace gases networking: Volatile organic carbon and nitrogen oxides, Deliverable D4.9, Final SOPs for VOCs measurements, Final Version, available at: http:// fp7.actris.eu/language/en-GB/Home.aspx (last access: 28 May 2018), 30 September 2014.

Conte, A., Landoni, C., Manini, P., Rabellino, L., and Riddle, S.: Moisture and Hydrocarbon Management for EUVL Tools: Ultra High Vacuum and Purge Gas Purification Solutions, International Symposium on Extreme Ultraviolet Lithography 2008, Lake Tahoe, USA, http://sematech.org/meetings/archives/litho/ 8285/poster/OC-P08-Landoni.pdf (last access: 28 May 2018), 2008.

Debono, O., Thévenet, F., Gravejat, P, Hequet, V., Raillard, C., Le Coq, L., and Locoge, N.: Gas phase photocatalytic oxidation of decane at ppb levels: removal kinetics, reaction intermediates and carbon mass balance, J. Photochem. Photobiol. A, 258, 1729, 2013.

Demichelis, A., Sassi, G., Lecuna, M., and Sassi, M. P.: Molar fraction stability in dynamic preparation of reference trace gas mixtures, IET Sci. Meas. Technol., 10, 414-419, https://doi.org/10.1049/iet-smt.2015.0051, 2016.

Haerri, H. P.: Trace gas analysis for the evaluation of zero air generators, Accredit. Qual. Assur., 14, 647-654, 2009.

Heck, R. M., Farrauto, R. J., and Gulati, S. T.: Catalytic air pollution control: commercial technology, John Wiley \& Sons, New Jersey, USA, 2009.

Hoerger, C. C., Claude, A., Plass-Duelmer, C., Reimann, S., Eckart, E., Steinbrecher, R., Aalto, J., Arduini, J., Bonnaire, N., Cape, J. N., Colomb, A., Connolly, R., Diskova, J., Dumitrean, P., Ehlers, C., Gros, V., Hakola, H., Hill, M., Hopkins, J. R., Jäger, J., Junek, R., Kajos, M. K., Klemp, D., Leuchner, M., Lewis, A. C., Locoge, N., Maione, M., Martin, D., Michl, K., Nemitz, E., O'Doherty, S., Pérez Ballesta, P., Ruuskanen, T. M., Sauvage, S., Schmidbauer, N., Spain, T. G., Straube, E., Vana, M., Vollmer, M. K., Wegener, R., and Wenger, A.: ACTRIS non-methane hydrocarbon intercomparison experiment in Europe to support WMO GAW and EMEP observation networks, Atmos. Meas. Tech., 8, 2715-2736, https://doi.org/10.5194/amt-8-2715-2015, 2015.

Huang, Y, Ho, S. S. H., Lu, Y., Niu, R., Xu, L., Cao, J., and Lee, S.: Removal of indoor volatile organic compounds via photocatalytic oxidation: a short review and prospect, Molecules, 21, 56, 1-20, 2016.

ISO 6145-7: Gas analysis - Preparation of calibration gas mixtures using dynamic volumetric methods, Part 7: Thermal mass-flow controllers, 2009.

ISO 6145-8: Gas analysis - Preparation of calibration gas mixtures using dynamic volumetric methods, Part 8: Diffusion method, 2005.

ISO 6145-10: Gas analysis - Preparation of calibration gas mixtures using dynamic volumetric methods, Part 10: Permeation method, 2002.

International Union of Pure and Applied Chemistry (IUPAC): Nomenclature in Evaluation of Analytical Methods Including 
Detection and Quantification Capabilities, prepared by: Currie, L. A., Pure Appl. Chem., 67, 1699-1723, 1995.

Liotta, L. F.: Catalytic oxidation of volatile organic compounds on supported noble metals, Appl. Catalys. B, 100, 403-412, 2010.

Miñarro, M. D., Brewer, P. J., Brown, R. J. C., Persijn, S., van Wijk, J., Nieuwenkamp, G., Baldan, A., Kaiser, C., Sutour, C., Macéé, T., Škundrić, N., and Tarhan, T.: Zero gas reference standards, Analyt. Meth., 8, 3014-3022, 2016.

Plass-Duelmer, C., Michl, K., Ruf, R., and Berresheim, H.: $\mathrm{C}_{2}-$ $\mathrm{C}_{8}$ Hydrocarbon measurement and quality control procedures at the Global Atmosphere Watch Observatory Hohenpeissenberg, J. Chromatogr. A, 953, 175-197, 2002.
Sircar, S., Golden, T. C., and Rao, M. B.: Activated carbon for gas separation and storage, Carbon, 34, 1-12, 1996.

Van Osdell, D. W., Owen, M. K., Jaffe, L. B., and Sparks, L. E.: VOC removal at low contaminant concentrations using granular activated carbon, J. Air Waste Manage. Assoc., 46, 883-890, 1996.

WMO: A WMO/GAW Expert Workshop on Global Long-Term Measurements of Volatile Organic Compounds (VOCs), GAW Report No. 171, WMO, Geneva, Switzerland, 2007. 